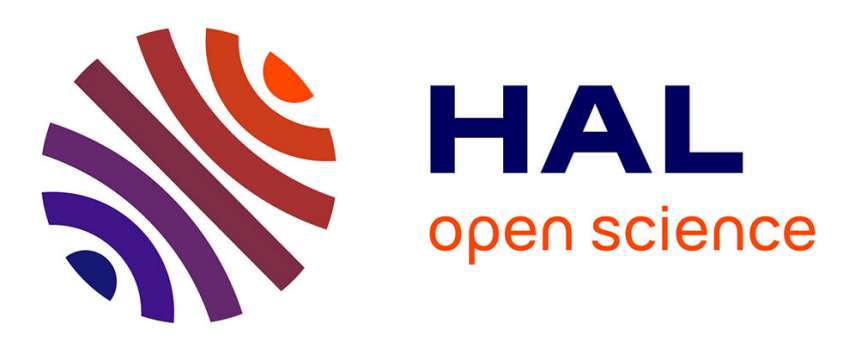

\title{
A note on the decomposability of inequality measures
}

\author{
Frederic Chantreuil, Sebastien Courtin, Kévin Fourrey, Isabelle Lebon
}

\section{To cite this version:}

Frederic Chantreuil, Sebastien Courtin, Kévin Fourrey, Isabelle Lebon. A note on the decomposability of inequality measures. Social Choice and Welfare, 2019, 53 (2), pp.283-298. 10.1007/s00355-01901183-9 . halshs-02294331

\section{HAL Id: halshs-02294331 https://shs.hal.science/halshs-02294331}

Submitted on 21 Oct 2019

HAL is a multi-disciplinary open access archive for the deposit and dissemination of scientific research documents, whether they are published or not. The documents may come from teaching and research institutions in France or abroad, or from public or private research centers.
L'archive ouverte pluridisciplinaire HAL, est destinée au dépôt et à la diffusion de documents scientifiques de niveau recherche, publiés ou non, émanant des établissements d'enseignement et de recherche français ou étrangers, des laboratoires publics ou privés. 


\title{
A note on the decomposability of inequality measures*
}

\author{
Frédéric Chantreuil, Sébastien Courtin, Kevin Fourrey and Isabelle Lebon ${ }^{\dagger}$
}

November 20, 2018

\begin{abstract}
We propose a decomposition of inequality measures. By taking the example of the decomposition of income inequality by components, we show that this decomposition fits the definition of two elements: the sum of pure marginal contributions of income components and the sum of the pairwise interactions of all income components. This decomposition relies on the Shapley function and remains valid for a decomposition by subgroups and by components.
\end{abstract}

Keywords: Interactions, Inequality decomposition, Shapley

JEL Codes: C71, D63.

\section{Introduction}

The literature in the field of inequality measures has developed specific techniques to solve the problem of decomposition of aggregate inequality into some relevant components contributions. The issue to which this kind of analysis has been applied generally falls into two broad cases. ${ }^{1}$ The first one considers cases in which the concerned population is divided into different subgroups (see for instance the pioneer

${ }^{*}$ The authors thank also two anonymous reviewers and one associate editor for their useful comments. This research has been developed within the CoCoRICo-CoDEC research program (ANR-14CE24-0007-02).

${ }^{\dagger}$ Normandie Univ, Unicaen, CREM, UMR CNRS 6211, France, and TEPP-CNRS

${ }^{1}$ Note that Chantreuil and Lebon [2015] proposed a third case, which is the decomposition by individuals' attributes. 
articles of Bourguignon [1979], Cowell [1980], Shorrocks [1980, 1984, 1988]). The second case applies when the targeted variable is the sum of the various components that constitute the source of its value (see Shorrocks [1982]).

As pointed out by Cowell and Fiori [2011], these two main strands of decomposition by subgroup and by source are often treated as entirely separate from one another and few attempts have been made to construct a single framework for both principal types of decomposition. A notable exception are the decompositions proposed by Chantreuil and Trannoy [2011, 2013] and Shorrocks [2013] which determine the importance of each component (every source or population subgroup) to explain the overall inequality. These decompositions are all based on the basic idea that a casual approach for decomposing inequality consists of assigning to every components its direct marginal contribution to overall inequality, i.e. the difference between overall inequality and the inequality if we removed the inequality attributable to this component. Unfortunately, this simple view leads to marginal contributions that may not add up to the amount of total inequality that needs to be decomposed. In other words, the marginalist view cannot explain all inequality, resulting in a nonconsistent decomposition of the overall inequality.

In the context of inequality games, Chantreuil and Trannoy $[2011,2013]$ and Shorrocks [2013] show that the Shapley value (Shapley [1953]) is the only way to reconcile the marginalist approach with the full explanation of inequality decomposition in the case of income inequality. The contribution of a given component to the overall inequality is thus measured by the Shapley value of this component in the corresponding inequality game.

Despite its attractiveness, we dispute the validity of the implicit interpretation of the component's Shapley value as its contribution to the overall inequality by defending the idea that the non-consistency of the marginalist view is due to its inability to take into account the links between the components. Indeed, one can argue that the components are connected, or at least not independent from each other. The nonconsistency of the marginalist view is simply a consequence of not accounting for those interactions.

We propose a new interpretation, inspired by the literature on non-additive measures, that allows us to account for those links. To our knowledge, the notion of interaction was first developed by Owen $[1972]^{2}$. Consider a set of individuals re-

\footnotetext{
${ }^{2}$ See also Murofushi and Soneda [1993], Grabish [1996, 1997], Grabish et al. [2000] and Kojadinovic [2002, 2004, 2005].
} 
ceiving an income from two components, for instance labor income and capital income. By looking for potential interactions between these two components, one can compare the level of the overall income inequality to the sum of the labor income inequality and the capital income inequality. The sign of this difference gives us the nature of the interaction between the two components. In the case of a positive sign, the components interact negatively in the sense that their effects are cumulative, leading to a situation in which the total inequality exceeds the sum of the inequalities measured for each component. In the case of a negative sign, the components interact positively in the sense that they compensate each other resulting in a situation in which the income inequality is lower than the sum of the inequalities by component. In the third case, if there is no difference, the components do not interact.

When the studied problems involve three components or more, as is the case with the examples used in this article, the highlighting of the pure marginal contributions of each of these components brings out the interactions between them two by two. Indeed, the difference between the decomposition before and after the application of the equalizing measure is translated only by pairwise interactions. Moreover, it appears that the theoretical results meet the needs of the empirical analysis as the interactions between two components are easily interpretable, whereas those that connect three components or more would not be in most cases.

Within this framework, we show that the importance of an income component equals the sum of its direct marginal contribution and a weighted sum of its pairwise interactions with all other components. This result helps us to derive a decomposition of inequality indices into two elements: the sum of pure marginal contributions of income components and the sum of the pairwise interactions of all income components. The outline of the remaining part of the paper is as follows. The next section introduces some notations and presents the considered concept of interactions. Section 3 presents our main results, while the last section concludes.

\section{Inequality game}

In the following, we will focus on the decomposition of income inequality by income components. Nevertheless, our approach remains valid for the decomposition of inequality by population subgroups since it is independent from the case studied. 
We thus consider a set of individuals $N:=\{1, \ldots, n\}$, with $n \geq 2$, and a set of income components $M:=\{1, . ., m\}$, with $m \geq 2$. Generic individuals will be denoted by $a, b$ while income components will be denoted by $i, j$. Any non-empty subset of income components $S$ is called a coalition and $\mathcal{M}$ is the set of non-empty subsets of income components. We consider a situation $x:=\left[x_{a}^{i}\right]$, where $x_{a}^{i} \geq 0$ is the amount of each income component $i$ received by individual $a$. $x$ can be viewed as an $n \times m$ matrix, where row $a$ indicates the amount of each income component received by individual $a$, and column $i$ indicates the distribution of component $i$ between all $n$ individuals. Let us denote by $\mathcal{X}:=\left\{x \mid x_{a}^{i} \geq 0 . \forall a, i\right\}$ the set of all situations $x$. With respect to a given situation $x$, the distribution of income by component $i$ is given by $x^{i}:=\left(x_{1}^{i}, \ldots, x_{n}^{i}\right)^{T}$, with $T$ the transposed vector. The aggregate distribution is $X:=\sum_{i \in M} x^{i}$ while the corresponding mean income is given by $\mu(X):=\frac{1}{n} \sum_{i \in M} x^{i}$. Likewise, the aggregate distribution for the set $S$ of components is $X^{S}:=\sum_{i \in S} x^{i}$ and $\mu\left(X^{S}\right):=\frac{1}{n} \sum_{i \in S} x^{i}$ is the corresponding mean income.

The inequality of a situation $x$ is measured by a function $I: \mathbb{R}^{n} \rightarrow[0,1]$ such that $I(X)=0$ for an equal income distribution. Moreover, $I($.$) is supposed to be scaled in-$ variant, that is $I(x)=I(\alpha x), \forall \alpha \in \mathbb{R}_{+}$. For a given inequality index $I$, the distribution of income by components helps us to build a distribution of income among subsets of components, namely an application $Y: \mathcal{M} \rightarrow \mathbb{R}^{m}$, such that $Y(\emptyset)=0$ and for all $S \in \mathcal{M}$

$$
Y(S, \lambda)=\left(\sum_{i \in S} x_{1}^{i}+\lambda, \ldots, \sum_{i \in S} x_{n}^{i}+\lambda\right)
$$

with $\lambda \in \mathbb{R}_{+}$.

Equation (1) leads to the definition of an $\lambda$-inequality game, that is a pair $\left(M, V_{I}\right)$, where $V_{I}$ is a function defined on all subsets $S \in \mathcal{M}$, such that $V_{I}(\emptyset)=0, V_{I}(M)=I(x)$ and for all $S, V_{I}(S)=I(Y(S))$. Let $\mathcal{V}$ the set of all $\lambda$-inequality games.

Depending on $\lambda$, Chantreuil and Trannoy [2011] define different inequality games. They first introduce zero income inequality games $(\lambda=0)$, in which the value of the function $V_{I}$, for some subset of components $S$ is simply the value of the inequality index if the individuals receive nothing from components not included in $S$. A second game, the equalized inequality game, considers that the value for some subset $S$ is given by the value of the inequality index when we have equalized the income for all sources not in $S$, i.e. $\lambda=\mu(X)-\mu\left(X^{S}\right)$. 
The article proposes two different approaches to drop inequalities coming from sources. Their values for all individuals are replaced either by their mean $(\lambda=$ $\left.\mu(X)-\mu\left(X^{S}\right)\right)$ or by zero $(\lambda=0)$. In both cases, values are replaced by a constant, that is why the article does not consider absolute index, as they are invariant to a translation.

In practice, the choice of a zero income inequality game or an equalized income inequality game depends primarily on a logic related to the nature of the question studied. In the anticipation of the effects of an economic measure, an equalized income game could, for example, be used to judge the effects on the total inequality of the alignment of premiums paid to employees of a particular organization, as the zero income game could, for example, be used to simulate the effects of the abolition of family allowances. In this perspective, the zero income game, beyond the theoretical result, has a very real interest in dealing with concrete economic questions. In either case, it might be objected that it would be as simple to directly calculate the future level of inequality by rebuilding the income distribution that would result from the measure, but the proposed decomposition also makes it possible to understand and to analyse the causes of the result. It is indeed the interactions with the other components that lead to the equalization of the premiums or the abolition of the allowances to increase or to decrease the total inequality according to which the inequalities resulting from the various elements of the income tend to offset or cumulate each other. These phenomena can be appraised and evaluated, component by component, through the Shapley decomposition.

Example 1. Let us consider the situation $x$ described in Table 1, where three income components $(i, j, h)$ and three individuals $(a, b, c)$ are taken into account. We define the $\lambda$-inequality games and compute income inequality using the Gini index.

\begin{tabular}{l|ccc}
\multicolumn{5}{c}{ Table 1: Income distributions } \\
\hline & Component $i$ & Component $j$ & Component $h$ \\
\hline Individual $a$ & 3 & 8 & 9 \\
Individual $b$ & 5 & 10 & 15 \\
Individual $c$ & 7 & 12 & 21
\end{tabular}

From this situation $x$, the associated $\lambda$-inequality game is defined, for $\lambda=0$ (Table 3 ) and $\lambda=\mu(X)-\mu\left(X^{S}\right)$ (Table 2). 
Table 2: Equalized income inequality game

\begin{tabular}{c|ccccccc}
\hline & $i$ & $j$ & $h$ & $i+j$ & $i+h$ & $j+h$ & $i+j+h$ \\
\hline Individual $a$ & 28 & 28 & 24 & 26 & 22 & 22 & 20 \\
Individual $b$ & 30 & 30 & 30 & 30 & 30 & 30 & 30 \\
Individual $c$ & 32 & 32 & 36 & 34 & 38 & 38 & 40 \\
\hline & $V_{I}(i)$ & $V_{I}(j)$ & $V_{I}(h)$ & $V_{I}(i, j)$ & $V_{I}(i, h)$ & $V_{I}(j, h)$ & $V_{I}(i, j, h)$ \\
\hline Gini & 0.0296 & 0.0296 & 0.0889 & 0.0592 & 0.1185 & 0.1185 & 0.1481
\end{tabular}

Table 3: Zero income inequality game

\begin{tabular}{c|ccccccc}
\hline & $i$ & $j$ & $h$ & $i+j$ & $i+h$ & $j+h$ & $i+j+h$ \\
\hline Individual $a$ & 3 & 8 & 9 & 11 & 12 & 17 & 20 \\
Individual $b$ & 5 & 10 & 15 & 15 & 20 & 25 & 30 \\
Individual $c$ & 7 & 12 & 21 & 19 & 28 & 33 & 40 \\
\hline & $V_{I}(i)$ & $V_{I}(j)$ & $V_{I}(h)$ & $V_{I}(i, j)$ & $V_{I}(i, h)$ & $V_{I}(j, h)$ & $V_{I}(i, j, h)$ \\
\hline Gini & 0.1778 & 0.0889 & 0.1778 & 0.1185 & 0.1778 & 0.1422 & 0.1481
\end{tabular}

\section{Importance of an income component}

For each $\lambda$-inequality game $\left(M, V_{I}\right)$, an importance function $\operatorname{IMP}\left(M, V_{I}\right)$ is mapping that assigns a vector of real values representing the importance of each component $i$. Thus, a component reduces income inequality if its importance is negative, while a positive importance leads to an increase of income inequality.

\subsection{Marginal Contribution}

As pointed out in the introduction, a natural approach to evaluate this importance would proceed by assigning to every income component its pure marginal contribution to the overall inequality.

\section{Definition 1. The pure marginal contribution}

For a given $\lambda$-inequality game $\left(M, V_{I}\right)$, the pure marginal contribution (PMC) of the income component $i \in M$ is defined by:

$$
P M C_{i}\left(M, V_{I}\right)=V_{I}(M)-V_{I}(M \backslash\{i\})
$$

Definion 1 states that the pure marginal contribution of a given component $i$ is the difference between the value of the function $V_{I}$ for the entire set of income components and the value of the function $V_{I}$ for the entire set of income components 
when the component $i$ has a null distribution (i.e. for the zero inequality game) or an equalized distribution (i.e. for the equalized inequality game). This PMC can be positive or negative. In the first case, it means that if the component were no longer included in the coalition then income inequality would decrease, while if the PMC were negative, income inequality would increase.

Example 2. (Example 1 continued)

The previous equalized inequality game (Table 2) shows that the marginalist view may be consistent in explaining the overall income inequality. Indeed, as shown in Table 4, the sum of the pure marginal contribution equals the overall income inequality of the situation $x$ measured by the Gini index.

Table 4: Pure Marginal Contributions - Equalized Inequality Game

\begin{tabular}{cccc}
$P M C_{i}\left(M, V_{I}\right)$ & $P M C_{j}\left(M, V_{I}\right)$ & $P M C_{h}\left(M, V_{I}\right)$ & Sum \\
\hline 0.0296 & 0.0296 & 0.0889 & $0.1481=V_{I}(i, j, h)$
\end{tabular}

Nevertheless, this naive solution is problematic as it is not always consistent, regardless of the type of $\lambda$-inequality game. Table 5 illustrates that in the zero inequality game (defined in Table 3), $\sum_{i \in M} P M C_{i}\left(M, V_{I}\right) \neq V_{I}(M)=I(x)$.

Table 5: Pure Marginal Contributions - Zero Inequality Game

\begin{tabular}{cccc}
$P M C_{i}\left(M, V_{I}\right)$ & $P M C_{j}\left(M, V_{I}\right)$ & $P M C_{h}\left(M, V_{I}\right)$ & Sum \\
\hline 0.0059 & -0.0296 & 0.0296 & $0.0059 \neq V_{I}(i, j, h)$ \\
\hline
\end{tabular}

Therefore, decomposing income inequality with the marginalist approach may lead to an unsatisfactory result. Chantreuil and Trannoy $[2011,2013]$ and Shorrocks [2013] show that the Shapley value (Shapley [1953]) is the only function that solves the problem of non-consistency of the marginal contribution principle.

\section{Definition 2. The Shapley function}

For a given $\lambda$-inequality game $\left(M, V_{I}\right)$, with $s=|S|$, the Shapley function (SHAP) of a component $i \in M$ is given by:

$$
\operatorname{SHAP}_{i}\left(M, V_{I}\right)=\sum_{\substack{S \subseteq \mathcal{M} \\ i \notin \mathcal{S}}} \frac{(m-s-1) ! s !}{m !}\left[V_{I}(S \cup\{i\})-V_{I}(S)\right]
$$


Example 3. (Example 1 continued)

The importance of the components obtained with the Shapley function are given in Table 6.

Table 6: Importances

\begin{tabular}{c|cccc}
\hline & $\operatorname{SHAP}_{i}\left(M, V_{I}\right)$ & $\operatorname{SHAP}_{j}\left(M, V_{I}\right)$ & $\operatorname{SHAP}_{h}\left(M, V_{I}\right)$ & Sum \\
\hline Zero Game & 0.0662 & 0.0039 & 0.0780 & $0.1481=V_{I}(i, j, h)$ \\
\hline Equalized Game & 0.0296 & 0.0296 & 0.0889 & $0.1481=V_{I}(i, j, h)$
\end{tabular}

In both games, the sum of the importance of components $i, j$ and $h$, measured with the Shapley function, equals the overall inequality.

Note that in the equalized inequality game, the importances measured by the PMC (Table 4) and by the Shapley function are identical.

In the zero inequality game, the PMC of component $j$ (Table 5) is negative, which means that it should increase the overall inequality if the differences in income observed in its distribution are removed. Thus, component $j$ would decrease inequality.

However, the sign of the importance of component $j$ calculated with the Shapley function indicates that this component increases inequality.

This result raises the question of differences in the treatment of component $j$ to explain the overall inequality. What is the adequate approach to evaluate the importance of income components - the PMC approach or the Shapley decomposition approach? We argue that the appropriate process to implement such an evaluation is the Shapley function, not merely for its consistency property. In fact, the importance of a given component has to be assessed by taking into account the interactions between components.

\subsection{Interaction between components}

As pointed out in the introduction, the interaction between two income components depends on the difference between the inequality when two income components are combined and the sum of each component income inequality. Formally, for a $\lambda$-inequality game $\left(M, V_{I}\right)$ and for all pairs of income components $i, j \in M$, the interaction between $i$ and $j$ depends on the difference $V_{I}(\{i, j\})-V_{I}(\{i\})-V_{I}(\{j\})$. Nevertheless, the remaining income components belonging to $M$ should modify the interaction between components $i$ and $j$. To account for this phenomenon, Murofushi 
and Soneda [1993] introduced what Kojadinovic [2005] calls the interaction between components $i$ and $j$ in the presence of $T$, where $T$ is a subset of income components not containing $i$ and $j$. Taking into account that the interaction between two components $i \in M$ and $j \in M, i \neq j$ depends on the interaction between $i$ and $j$ in the presence of any possible subsets of other components, we can formally establish the interaction between two components as follows.

\section{Definition 3. Pairwise interaction}

For a given $\lambda$-inequality game $\left(M, V_{I}\right)$ and for all pairs of income components $i, j \in M$, $i \neq j$, the interaction between income components $i$ and $j$ in the presence of the subset of components $T \subseteq \mathcal{M}, i \notin T$ and $j \notin T$ is defined by:

$$
\left.\left.\operatorname{Int}(i, j, T)=V_{I}(\{i, j\} \cup T)-V_{I}(\{i\} \cup T)\right)-V_{I}(\{j\} \cup T)\right)+V_{I}(T)
$$

Example 4. (Example 1 continued)

We compute for each component all pairwise interactions, as defined by equation (4). The results are given in Table 7.

Table 7: Interactions

\begin{tabular}{c|cccc}
\hline Component $i$ & $\operatorname{Int}(i, j,\{\emptyset\})$ & $\operatorname{Int}(i, j,\{h\})$ & $\operatorname{Int}(i, h,\{\emptyset\})$ & $\operatorname{Int}(i, h,\{j\})$ \\
\hline Zero Game & -0.1481 & 0.0059 & -0.1778 & -0.0237 \\
Equalized Game & 0.0000 & 0.0000 & 0.0000 & 0.0000 \\
\hline Component $j$ & $\operatorname{Int}(j, i,\{\emptyset\})$ & $\operatorname{Int}(j, i,\{h\})$ & $\operatorname{Int}(j, h,\{\emptyset\})$ & $\operatorname{Int}(j, h,\{i\})$ \\
\hline Zero Game & -0.1481 & 0.0059 & -0.1244 & 0.0296 \\
Equalized Game & 0.0000 & 0.0000 & 0.0000 & 0.0000 \\
\hline Component $h$ & $\operatorname{Int}(h, i,\{\emptyset\})$ & $\operatorname{Int}(h, i,\{j\})$ & $\operatorname{Int}(h, j,\{\emptyset\})$ & $\operatorname{Int}(h, j,\{i\})$ \\
\hline Zero Game & -0.1778 & 0.0059 & -0.1244 & -0.0237 \\
Equalized Game & 0.0000 & 0.0000 & 0.0000 & 0.0000
\end{tabular}

In the zero inequality game, the pair of components $i$ and $j$ is characterized by a negative interaction when it is taken on its own. More formally, $\operatorname{Int}(i, j,\{\emptyset\})=0.1185-(0.1778+$ 0.0889), and, consequently, when considering components $i$ and $j$ together, the income inequality decreases.

However, adding the component $h$ to the pair of components $(i, j)$ changes things since the interaction bewteen $i$ and $j$ in the presence of $h$ becomes positive (the inequality increases). Note that the pair of components $i$ and $h$ is also characterized by a negative interaction, but adding the component $j$ to the pair of components $(i, h)$ does not change the sign of the 
interaction. Note that in this particular equalized inequality game, all pairwise interactions are null. This result can obviously be derived by the fact that the PMC approach and the Shapley function give equal importance to each component. This result may not hold for another example.

The previous example suggests accounting for all the possible combinations of pairwise interactions, leading to a new importance function.

\section{Definition 4. The Shapley function revisited}

For a given $\lambda$-inequality game $\left(M, V_{I}\right)$, the Shapley function revisited $\left(\overline{\operatorname{SHAP}_{i}}\left(M, V_{I}\right)\right)$ of an income component $i \in M$ is defined by:

$$
\overline{\operatorname{SHAP}_{i}}\left(M, V_{I}\right)=P M C_{i}-\sum_{\substack{j \in M \\ i \neq j}} I N T(i, j, T)
$$

With, INT $(i, j, T)=\sum_{\substack{T \subset \mathcal{M} \\ i, j \in T}} \frac{(m-t-2) !(t+1) !}{m !}(\operatorname{Int}(i, j, T)), m=|M|$ the number of components and $t=|T|$ the number of components in a coalition $T^{3}$.

For a given $\lambda$-inequality game $\left(M, V_{I}\right)$, definition 4 states that the importance of an income component equals the sum of its pure marginal contribution minus a weighted sum of all its pairwise interactions. Table 8 presents the six possible cases that may define the sign of the importance of components.

Table 8: Interactions

\begin{tabular}{l|c|c||c}
\hline & $P M C_{i}$ & $\sum_{\substack{j \in M \\
i \neq j}} I N T(i, j, T)$ & $\overline{\operatorname{SHAP}_{i}}\left(M, V_{I}\right)$ \\
\hline Case 1 & + & - & + \\
\hline Case 2 & - & + & - \\
\hline Case 3 & + & + & + \\
\hline Case 4 & + & + & - \\
\hline Case 5 & - & - & - \\
\hline Case 6 & - & - & +
\end{tabular}

\footnotetext{
${ }^{3}$ For instance, if there are two components $(m=2)$, the coalition considered to evaluate the interaction between $i$ and $j$ only includes these two components $(t=0)$. Thus, in this particular case, the weight of this interaction is $\frac{(2-0-2) !(0+1) !}{2 !}=\frac{0 ! 1 !}{2 !}=\frac{1}{2}$. Both components will have an importance equals to their PMC, plus half of value of their interaction.
} 
When the signs of the PMC and the interactions are opposite (cases 1 and 2), the importance of the component on inequality is reinforced, regardless of their relative magnitudes. When the signs are identical, the relative magnitude of the effects lead to the sign of the importance. In case 3, the PMC effect is larger than the interactions effects and the importance is thus positive. In case 4 , however, the level of the interactions does not counterbalance the PMC effect, leading to a negative importance. A symmetric situation appears in cases 5 and 6 .

Example 5. (Example 1 continued)

We can now compute for each component $\overline{\operatorname{SHAP}}\left(M, V_{I}\right)$.

Table 9: Importances

\begin{tabular}{c|cccc}
\hline Component $i$ & $P M C_{i}$ & $I N T(i, j, T)$ & $I N T(i, h, T)$ & $\overline{\operatorname{SHAP}_{i}}\left(M, V_{I}\right)$ \\
\hline Zero Game & 0.0059 & -0.0227 & -0.0376 & 0.0662 \\
Equalized Game & 0.0296 & 0.0000 & 0.0000 & 0.0296 \\
\hline Component $j$ & $P M C_{j}$ & $I N T(j, i, T)$ & $I N T(j, h, T)$ & $\overline{S_{H A P}}\left(M, V_{I}\right)$ \\
\hline Zero Game & -0.0296 & -0.0227 & -0.0108 & 0.0039 \\
Equalized Game & 0.0296 & 0.0000 & 0.0000 & 0.0296 \\
\hline Component $h$ & $P M C_{h}$ & $I N T(h, i, T)$ & $I N T(h, j, T)$ & $\overline{S_{H A P}}\left(M, V_{I}\right)$ \\
\hline Zero Game & 0.0296 & -0.0375 & -0.0109 & 0.0780 \\
Equalized Game & 0.0889 & 0.0000 & 0.0000 & 0.0889
\end{tabular}

For both types of income inequality games, the Shapley function revisited leads to the same results as the Shapley function. As was previously the case, since the interactions are null in the equalized game, the PMC of each component equals the Shapley function revisited.

We can now provide our main result.

\section{Proposition 1. Shapley revisited}

For a given $\lambda$-inequality game $\left(M, V_{I}\right)$, and for each component

$$
\overline{\operatorname{SHAP}_{i}}\left(M, V_{I}\right)=\operatorname{SHAP}\left(M, V_{I}\right)
$$

Proposition 1 states that the importance of a given component, measured by the Shapley function, can be expressed as the sum of its pure marginal contribution and a sum of the pairwise interactions of this component with all the other components. 
Proof. The proof of proposition 1 is given in the appendix.

From proposition 1, we can derive the following result.

Proposition 2. The decomposability of inequality measures

For a given $\lambda$-inequality game $\left(M, V_{I}\right)$, the inequality $V_{I}(M)=I(x)$ is decomposable as follows:

$$
I(x)=\sum_{i \in M} P M C_{i}-\sum_{i \in M} \sum_{\substack{j \in M \\ i \neq j}} I N T(i, j, T)
$$

Proof. The consistency of the Shapley function leads to the desired result.

Proposition 2 states that all inequality measures can be decomposed into two terms, namely the sum of the pure marginal contribution of all income components minus the sum of the weighted sums of all pairwise interactions of income components with all the other income components, and those for all components.

\section{Conclusion}

Many decomposition approaches have been proposed in the literature. The two first ones, the sub-population decomposition and the income sources decompositions, aimed to estimate the contribution of respectively each sub-population and each sources to inequality. Then, some multi-decompositions, based on mathematical arrangements, suggested to take into account at the same time group and source effects, even by taking into consideration temporal effects (Mussard and Savard [2012]). Alternatively, Shorrocks [2013] and Chantreuil and Trannoy [2013] have also proposed a multi-decomposition, based on the Shapley value. As the methods based on a mathematical arrangements, this approach proposed to take into account at the same time group and sources effects, by considering them at two different levels (through a hierarchical models based on the Owen value). Another approach also based on the Shapley value, proposed by Chantreuil and Lebon [2015], considers various groups effects (ex.: age, gender) at the same time by treating them as components. Thus, the Shapley decomposition of inequality index has three main 
advantages. First, it can take many dimensions of inequality. Then, the same approach can be applied to a large variety of inequality index. Finally, as it has been shown in this article, this approach can distinguish the pure marginal contribution of a component and its total contribution to inequality.

Indeed, we recall that the marginalist approach of the decomposition of an inequality index, however appealing, is not coherent because the sum of contributions will not always amount to the overall inequality. The Shapley decomposition is, on the contrary, consistent and differs from the marginalist approach accounting for the interactions between the components. By revisiting the Shapley function, we show that the importance of a given component, measured by the Shapley function, can be expressed as the difference between its pure marginal contribution and the sum of pairwise interactions that can be positive or negative. Hence, we obtain a new way to decompose all inequality measures determining the links between each pair of components and the impact of this relationship on the importance of a component. Considering these interactions seems crucial to evaluate the consequences of public policies that aimed at dropping inequalities, as the impact of eliminating inequalities from a component is modulated by its link with the others. In this perspective, there is no need to make an a priori choice between an equalized income inequality game and a zero income inequality game, one or the other applies depending on the type of envisaged measure.

\section{References}

F. Bourguignon. Decomposable income inequality measures. Econometrica, 47:901920, 1979.

F. Chantreuil and I. Lebon. Gender contribution to income inequality. Economics Letters, 133:27-32, 2015.

F. Chantreuil and A. Trannoy. Inequality decomposition values. Annals of Economics and Statistics, 101/102:6-29, 2011.

F. Chantreuil and A. Trannoy. Inequality decomposition values: the trade-off between marginality and efficiency. The Journal of Economic Inequality, 11:83-98, 2013. 
F. Cowell. Decomposable income inequality measures. Review of Economic Studies, 47:520-531, 1980.

F. Cowell and C.V. Fiori. Inequality decompositions: a reconciliation. The Journal of Economic Inequality, 9:509-528, 2011.

M. Grabish. The representation of importance and interaction of features by fuzzy measures. Pattern Recognition, 17:567-575, 1996.

M. Grabish. Alternative representations of discrete fuzzy measures for decision making. International Journal of Uncertainty Fuzziness Knowledge-Based Syst, 5:587-607, 1997.

M. Grabish, J.L. Marichal, and M. Roubens. Equivalent representations of set functions. Mathematical Operation Research, 25:157-178, 2000.

I. Kojadinovic. Modeling interaction phenomena using fuzzy measures: On the notions of interaction and independence. Fuzzy Sets Syst., 135:317-340, 2002.

I. Kojadinovic. Estimation of the weights of interacting criteria from the set of profiles by means of information-theoretic functionals. European Journal of Operational Research, 155:740-751, 2004.

I. Kojadinovic. An axiomatic approach to the measurement of the amount of interaction among criteria or players. Fuzzy Sets and Systems, 152(3):417-435, 2005.

T. Murofushi and S. Soneda. Techniques for reading fuzzy measures (iii): interaction index. Proceedings of the 9th, Fuzzy System Symposium, Saporo, Japan, pages 693696, 1993.

S. Mussard and L. Savard. The gini multi-decomposition and the role of Gini's transvariation: application to partial trade liberalization in the philippines. Applied Economics, 44(10):1235-1249, 2012.

G. Owen. Multilinear extension of games. Management Science, 18:64-79, 1972.

L. S. Shapley. A value for n-person games. In: Contrib. Theory of Games, II. In: Ann. Math. Stud., 28:307-317, 1953.

A. Shorrocks. The class of additively decomposable inequality measures. Econometrica, 48:613-625, 1980. 
A. Shorrocks. Inequality decomposition by factor component. Econometrica, 50: 193-211, 1982.

A. Shorrocks. Inequality decomposition by population subgroups. Econometrica, 51: 1369-1385, 1984.

A. Shorrocks. Aggregation issues in inequality measurement. In Eichhorn W. (ed) Measurement in Economics, Physica-Verlag, New York, 1988.

A. Shorrocks. Decomposition procedures for distributional analysis: a unified framework based on the shapley value. The Journal of Economic Inequality, 11: 99-126, 2013. 


\section{Appendix: Proof of Proposition 1}

Proof. For a given $\lambda$-inequality game $\left(M, V_{I}\right)$ and a component $i \in M$. Its corresponding Shapley function and Shapley function revisited are given by $\operatorname{SHAP}\left(M, V_{I}\right)$ and $\overline{\operatorname{SHAP}_{i}}\left(M, V_{I}\right)$, respectively.

First note that $S H A P_{i}\left(M, V_{I}\right)=\left(\sum_{\substack{S \subseteq \mathcal{M} \\ i \notin S}} \frac{s !}{m \cdot(m-1) \ldots(m-s)} V_{I}(S \cup\{i\})\right)-\left(\sum_{\substack{S \subseteq \mathcal{M} \\ i \notin S}} \frac{s !}{m \cdot(m-1) \ldots(m-s)} V_{I}(S)\right)$ $=\alpha-\beta$

In the same way, we have $\overline{\operatorname{SHAP}}\left(M, V_{I}\right)$

$$
\begin{aligned}
&=\left(V_{I}(M)-\sum_{j \neq i} \sum_{\substack{T \subseteq \mathcal{M} \\
i, j \notin T}} \frac{(m-t-2) !(t+1) !}{m !}\left[V_{I}(T \cup\{i j\})-V_{I}(T \cup\{i\})\right]\right) \\
&-\left(V_{I}(M \backslash\{i\})+\sum_{j \neq i} \sum_{\substack{T \subseteq \mathcal{M} \\
i, j \notin T}} \frac{(m-t-2) !(t+1) !}{m !}\left[V_{I}(T)-V_{I}(T \cup\{j\})\right]\right) \\
&=\left(V_{I}(M)-\sum_{j \neq i} \sum_{\substack{T \subseteq \mathcal{M} \\
i, j \notin T}} \frac{(m-t-2) !(t+1) !}{m !} V_{I}(T \cup\{i j\})+\sum_{j \neq i} \sum_{\substack{T \subseteq \mathcal{M} \\
i, j \notin T}} \frac{(m-t-2) !(t+1) !}{m !} V_{I}(T \cup\{i\})\right)- \\
&\left.V_{I}(M \backslash\{i\})+\sum_{j \neq i} \sum_{\substack{T \subseteq \mathcal{M} \\
i, j \notin T}} \frac{(m-t-2) !(t+1) !}{m !} V_{I}(T)-\sum_{j \neq i} \sum_{\substack{T \subseteq \mathcal{M} \\
i, j \notin T}} \frac{(m-t-2) !(t+1) !}{m !} V_{I}(T \cup\{j\})\right) \\
&=
\end{aligned}
$$

We will prove that $\alpha=\alpha^{\prime}$ and $\beta=\beta^{\prime}$ and therefore, $\operatorname{SHAP}_{i}\left(M, V_{I}\right)=\overline{\operatorname{SHAP}}\left(M, V_{I}\right)$.

First step: $\alpha=\alpha^{\prime}$

Let $\alpha^{\prime}=V_{I}(M)-A+B$, with

$$
A=\sum_{j \neq i} \sum_{\substack{T \subseteq \mathcal{M} \\ i, j \notin T}} \frac{(m-t-2) !(t+1) !}{m !} V_{I}(T \cup\{i j\})
$$

and

$$
B=\sum_{j \neq i} \sum_{\substack{T \subseteq \mathcal{M} \\ i, j \notin T}} \frac{(m-t-2) !(t+1) !}{m !} V_{I}(T \cup\{i\})
$$




\section{i) Consider first $A$}

Let $K=T \cup\{j\}$ with $|K|=k=t+1$. Then $K \subseteq M$ and $i \notin K$.

Therefore $A=\sum_{\substack{K \subseteq \mathcal{M} \\ i \notin K}} \sum_{j \in K} \frac{(m-(k-1)-2) !((k-1)+1) !}{m !} V_{I}(K \cup\{i\})$.

Moreover $A=\sum_{K \subseteq \mathcal{M}} k \frac{(m-(k-1)-2) !((k-1)+1) !}{m !} V_{I}(K \cup\{i\})$ since $j \in K$.

Thus, $A=\sum_{\substack{K \subseteq \mathcal{M} \\ i \notin K}} \frac{k \notin(m-1) \ldots(m-k)}{m \cdot(m-1} V_{I}(K \cup\{i\})$.

ii) Now consider $V_{I}(M)-A$

This is equivalent to $V_{I}(M)-\sum_{\substack{K \subseteq \mathcal{M} \\ i \in K}} \frac{k \cdot k !}{m \cdot(m-1) \ldots(m-k)} V_{I}(K \cup\{i\})$

$$
\begin{aligned}
& =V_{I}(M)-\frac{(m-1) \cdot(m-1) !}{m \cdot(m-1) \ldots(m-(m-1))} V_{I}(M)-\sum_{\substack{K \subset \mathcal{M} \\
i \notin K}} \frac{k \cdot k !}{m \cdot(m-1) \ldots(m-k)} V_{I}(K \cup\{i\}) \\
& =V_{I}(M)-\sum_{\substack{K=M \\
i \notin K}} \frac{(m-1) \cdot(m-1) !}{m !} V_{I}(M)-\sum_{\substack{K \subset \mathcal{M} \\
i \notin K}} \frac{k . k !}{m \cdot(m-1) \ldots(m-k)} V_{I}(K \cup\{i\}) \\
& =V_{I}(M)-\frac{(m-1)}{m} V_{I}(M)-\sum_{\substack{K \subset \mathcal{M} \\
i \notin K}} \frac{k . k !}{m \cdot(m-1) \ldots(m-k)} V_{I}(K \cup\{i\}) \\
& =\frac{1}{m} V_{I}(M)-\sum_{\substack{K \subset \mathcal{M} \\
i \notin K}} \frac{k \cdot k !}{m \cdot(m-1) \ldots(m-k)} V_{I}(K \cup\{i\})
\end{aligned}
$$

iii) Consider now $B$

Let $K^{\prime}=T$ with $\left|K^{\prime}\right|=k^{\prime}=t$. Since $j \notin K^{\prime}, B=\sum_{\substack{K^{\prime} \subseteq \mathcal{M} \\ i, j \notin K^{\prime}}}\left(m-k^{\prime}-1\right) \frac{\left(m-k^{\prime}-2\right) !\left(k^{\prime}+1\right) !}{m !} V_{I}\left(K^{\prime} \cup i\right)$.

Therefore $B=\sum_{\substack{K^{\prime} \subseteq \mathcal{M} \\ i, j \notin K^{\prime}}} \frac{\left(k^{\prime}+1\right) !}{m \cdot(m-1) \ldots\left(m-k^{\prime}\right)} V_{I}\left(K^{\prime} \cup i\right)=\sum_{\substack{K \subseteq \mathcal{M} \\ i \notin K}} \frac{(k+1) !}{m \cdot(m-1) \ldots(m-k)} V_{I}(K \cup\{i\})$.

iv) Finally, $V_{I}(M)-A+B=\frac{1}{m} V_{I}(M)-\sum_{\substack{K \subset \mathcal{M} \\ i \in K}} \frac{k . k !}{m \cdot(m-1) \ldots(m-k)} V_{I}(K \cup\{i\})$

$$
\begin{aligned}
& +\sum_{\substack{K \subset \mathcal{M} \\
i \notin K}} \frac{(k+1) !}{m \cdot(m-1) \ldots(m-k)} V_{I}(K \cup\{i\}) . \\
& \text { It follows that } V_{I}(M)-A+B=\frac{1}{m} V_{I}(M)+\sum_{\substack{K \subset \mathcal{M} \\
i \notin K}} \frac{(k+1) !-k . k !}{m \cdot(m-1) \ldots(m-k)} V_{I}(K \cup\{i\}) \\
& =\frac{1}{m} V_{I}(M)+\sum_{\substack{K \subset \mathcal{M} \\
i \notin K}} \frac{(k+1) !-k . k !}{m \cdot(m-1) \ldots(m-k)} V_{I}(K \cup\{i\})
\end{aligned}
$$




$$
\begin{aligned}
& =\frac{1}{m} V_{I}(M)+\sum_{\substack{K \subset \mathcal{M} \\
i \notin K}} \frac{k !}{m \cdot(m-1) \ldots(m-k)} V_{I}(K \cup\{i\}) \\
& =\sum_{\substack{K \subseteq \mathcal{M} \\
i \notin K}} \frac{k !}{m \cdot(m-1) \ldots(m-k)} V_{I}(K \cup\{i\}) \\
& =\alpha
\end{aligned}
$$

Second step: $\beta=\beta^{\prime}$

Let $\beta^{\prime}=V_{I}(M \backslash\{i\})+C-D$, with

$$
C=\sum_{j \neq i} \sum_{\substack{T \subseteq \mathcal{M} \\ i, j \notin T}} \frac{(m-t-2) !(t+1) !}{m !} V_{I}(T)
$$

and

$$
D=\sum_{j \neq i} \sum_{\substack{T \subseteq \mathcal{M} \\ i, j \notin T}} \frac{(m-t-2) !(t+1) !}{m !} V_{I}(T \cup\{j\})
$$

i) Firstly, we investigate $D$

Let $L=T \cup\{j\}$ with $|L|=l=t+1$. Then $L \subseteq \mathcal{M}, i \notin L$. Therefore $D=\sum_{\substack{L \subseteq \mathcal{M} \\ i \notin L}} \sum_{j \neq i} \frac{(m-(l-1)-2) !((l-1)+1) !}{m !} V_{I}(L)$. Moreover $D=\sum_{\substack{L \subseteq \mathcal{M} \\ i \notin L}} l \frac{(m-(l-1)-2) !((l-1)+1) !}{m !} V_{I}(L)$ since $j \in L$. Finally $D=\sum_{\substack{L \subseteq \mathcal{M} \\ i \notin L}} \frac{l . l !}{m \cdot(m-1) \ldots(m-l)} V_{I}(L)$.

ii) Secondly, we consider $V_{I}(M \backslash\{i\})-D$. This is equal to $V_{I}(M \backslash\{i\})-\sum_{L \subseteq \mathcal{M}} \frac{l . l !}{m .(m-1) \ldots(m-l)} V_{I}(L)$

$=V_{I}(M \backslash\{i\})-\frac{(m-1) \cdot(m-1) !}{m \cdot(m-1) \ldots(m-(m-1))} V_{I}(M \backslash\{i\})-\sum_{\substack{L \subset \mathcal{M} \\ i \notin L}} \frac{l . l !}{m \cdot(m-1) \ldots(m-l)} V_{I}(L)$

$=V_{I}(M \backslash\{i\})-\frac{(m-1)}{m} V_{I}(M \backslash\{i\})-\sum_{\substack{L \subset \mathcal{M} \\ i \notin L}} \frac{l . l !}{m \cdot(m-1) \ldots(m-l)} V_{I}(L)$

$=\frac{1}{m} V_{I}(M \backslash\{i\})-\sum_{\substack{L \subset \mathcal{M} \\ i \notin L}} \frac{l . l !}{m \cdot(m-1) \ldots(m-l)} V_{I}(L)$

iii) Thirdly, we study $C$

Let $L^{\prime}=T$ with $\left|L^{\prime}\right|=l^{\prime}=t$. Then $L^{\prime} \subseteq \mathcal{M}, i, j \notin L^{\prime}$. Since $j \notin L^{\prime}, C=\sum_{\substack{L^{\prime} \subseteq \mathcal{M} \\ i, j \notin L^{\prime}}}\left(m-l^{\prime}-\right.$ 1) $\frac{\left(m-l^{\prime}-2\right) !\left(l^{\prime}+1\right) !}{m !} V_{I}\left(L^{\prime}\right)$. Therefore $C=\sum_{\substack{L^{\prime} \subseteq \mathcal{M} \\ i, j \notin L^{\prime}}} \frac{\left(l^{\prime}+1\right) !}{m \cdot(m-1) \ldots\left(m-l^{\prime}\right)} V_{I}\left(L^{\prime}\right)$ 
$=\sum_{\substack{L \subseteq \mathcal{M} \\ i \notin L^{\prime}}} \frac{(l+1) !}{m \cdot(m-1) \ldots(m-l)} V_{I}(L)$.

iv) To conclude, it follows that

$$
\begin{aligned}
& V_{I}(M \backslash\{i\})+C-D=\frac{1}{m} V_{I}(M)+\sum_{\substack{L \subset \mathcal{M} \\
i \notin L}} \frac{(l+1) !}{m \cdot(m-1) \ldots(m-l)} V_{I}(L)-\sum_{\substack{L \subset \mathcal{M} \\
i \notin L}} \frac{l . l !}{m \cdot(m-1) \ldots(m-l)} V_{I}(L) . \\
& =\frac{1}{m} V_{I}(M \backslash\{i\})+\sum_{\substack{L \subset \mathcal{M} \\
i \notin L}} \frac{(l+1) !-l . l !}{m \cdot(m-1) \ldots(m-l)} V_{I}(L)=\frac{1}{m} V_{I}(M \backslash\{i\})+\sum_{\substack{L \subset \mathcal{M} \\
i \notin L}} \frac{l !}{m \cdot(m-1) \ldots(m-l)} V_{I}(L) \\
& =\sum_{\substack{L \subseteq \mathcal{M} \\
i \notin L}} \frac{l !{ }^{m}}{m \cdot(m-1) \ldots(m-l)} V_{I}(L)=\beta
\end{aligned}
$$

